cases being that this value may actually be attained in the latter case for one or more special values of $\theta .^{*}$

Finally, it may be observed that the values of the so-called absolute minima for the cases where area may be passed over four, five, six, $\cdots$ times are respectively $\frac{1}{4} l^{2} \theta, \frac{1}{6} l^{2} \theta, \frac{1}{6} l^{2} \theta, \cdots$. The consideration of these cases, however, on the geometrical side again presents serious difficulties, but tends to the opinion, as in the case of triplication, that in general the smallest area that can be swept over by any actual movement of angle $\theta$ is $\frac{1}{2} l^{2} \theta$ rather than any of these smaller values.

UNIVERSITY OF MichigAN.

\title{
CONVERGENCE OF SEQUENCES OF LINEAR OPERATIONS $\dagger$
}

BY T. H. HILDEBRANDT.

Let $U_{n}$ be a sequence of linear continuous operations on the class $F$ of functions $f$, continuous on the interval $(a, b)$, i.e., suppose that every $U$ satisfies the two conditions:

$$
U\left(c_{1} f_{1}+c_{2} f_{2}\right)=c_{1} U\left(f_{1}\right)+c_{2} U\left(f_{2}\right)
$$

for every pair of constants $\left(c_{1}, c_{2}\right)$ and every pair of functions $\left(f_{1}, f_{2}\right)$ of the class $F$;

(2) There exists a constant $M$ depending on $U$ such that if $N f$ is the maximum value of $|f|$ on $(a, b)$ then

$$
|U(f)| \leqq M N f \text {. }
$$

The greatest lower bound of all possible values $M$ might be called the modulus of $U$.

* Thus, in case $\theta=\pi$ and triplication is allowed, the corresponding value $\frac{1}{2} l^{2} \pi$ may be attained as follows: Construct the hypocycloid of three cusps obtained by rolling the circle of radius $\frac{1}{2} l$ within the circle of radius $\frac{3}{2} l$ and let the given segment (of length $2 l$ ) move so as to be always tangent to this curve and yet be everywhere entirely within it. The resulting area swept over as $\theta$ passes from 0 to $\pi$ is entirely triplicated, as is well known, and is equal to the amount above stated, $\frac{1}{2} l^{2} \pi$. See, for example, F. Gomes Teixeira, Traité des Courbes Spéciales Remarquables Planes et Gauches, vol. II, p. $193 . \quad$ (Coïmbre, 1909.)

$\dagger$ Presented to the Society, September 4, 1919. 
Helly* has shown that a necessary condition that $\lim _{n} U_{n}$ exists for every $f$ of $F$ is that the $U_{n}$ be uniformly bounded, i.e., that there exist a constant $M$ independent of $n$ such that

Then

$$
\left|U_{n}(f)\right| \leqq M N f
$$

$$
\lim _{n} U_{n}(f)=U(f),
$$

where $U(f)$ is a linear continuous operation whose modulus is less than or equal to $M$. In order to obtain sufficient conditions, it is necessary to make use of the classical theorem of Riesz, that every linear continuous operation on the class $F$ is expressible in the form of the Stieltjes integral, $\int f d \alpha$, where $\alpha$ is of bounded variation. If $\alpha$ is regular, i.e., such that at every point $x$ on $(a, b) \alpha(x)$ lies between $\alpha(x-0)$ and $\alpha(x+0)$, which can always be assumed to be the case without changing the value of the integral (and we shall restrict ourselves to this case), then the total variation $(\mathcal{S}|d \alpha|)$ of $\alpha$ is exactly the modulus of $U$, so that Helly's condition would be, that there exists a constant $M$ independent of $n$ such that

$$
\mathcal{S}\left|d \alpha_{n}\right|<M,
$$

for every $n$, i.e., the $\alpha_{n}$ are uniformly of bounded variation.

This condition is not sufficient. Helly $\dagger$ has shown that if (1) the $\alpha_{n}$ are uniformly of bounded variation and (2) there exists a function $\alpha$ of bounded variation such that

then

$$
\lim _{n} \alpha_{n}=\alpha \text { for every } x,
$$

$$
\lim _{n} \int f d \alpha_{n}=\int f d \alpha \text { for every } f \text { of } F .
$$

Bray $\ddagger$ has shown that a weaker second condition is that there exists a function of bounded variation $\alpha$ and a denumerable everywhere dense set of points: $x_{1}, \cdots, x_{m}, \cdots$ including $a$ and $b$, such that for every $x_{m}$

$$
\lim _{n} \alpha_{n}\left(x_{m}\right)=\alpha\left(x_{m}\right) .
$$

This condition is not necessary. It is the purpose of this note to derive necessary and sufficient conditions.

* Helly, Wiener Sitzungsberichte, vol. 121 (IIa) (1912), p. 268.

† Loc. cit., p. 288.

$\ddagger$ Annals of Mathematics, (2), vol. 20 (1919), p. 180. 
We derive first an additional necessary condition. Since the $\alpha_{n}$ are necessarily uniformly of bounded variation, it follows from a theorem by Helly* that the $\alpha_{n}$ are a compact set, i.e., there exists a subsequence $\alpha_{n_{m}}$ of the sequence $\alpha_{n}$ and a function $\alpha$ (necessarily of bounded variation) such that

Consequently

$$
\lim _{m} \alpha_{n_{m}}=\alpha \text { for every } x \text {. }
$$

$$
\lim _{m} \int f d \alpha_{n_{m}}=\int f d \alpha
$$

for every $f$ of $F$. Since $\lim _{n} \int f d \alpha_{n}$ exists, it follows that

$$
\lim _{n} \int f d \alpha_{n}=\int f d \alpha, \quad \text { or } \quad \lim _{n} \int f d\left(\alpha_{n}-\alpha\right)=0
$$

for every $f$ of $F$. Let

$$
\beta_{n}(x)=\alpha_{n}(x)-\alpha(x)-\alpha_{n}(a)+\alpha(a) .
$$

Then our condition becomes:

$$
\lim _{n} \int f d \beta_{n}=0
$$

for every $f$ of $F$. If we take $f=1$ then

$$
\lim _{n}\left[\beta_{n}(b)-\beta_{n}(a)\right]=\lim _{n} \beta_{n}(b)=0 .
$$

Again take $f=x$ for $a \leqq x \leqq \xi$ and $f=\xi$ for $\xi \leqq x \leqq b$. Then if we apply the integration by parts formula valid for Stieltjes integrals:

$$
\int f d \beta_{n}=\left.f \beta_{n}\right|_{a} ^{b}-\int \beta_{n} d f=\xi \beta_{n}(b)-\int_{a}^{\xi} \beta_{n} d x .
$$

Consequently, since $\lim _{n} \beta_{n}(b)=0$, we must have

$$
\lim _{n} \mathcal{S}_{a}^{x} \beta_{n} d x=0 \text { for every } x \text { of }(a, b)
$$

or the equivalent condition:

$\left(b^{\prime}\right) \lim _{n} \int_{a_{1}}^{b_{1}} \beta_{n} d x=0$ for every subinterval $\left(a_{1}, b_{1}\right)$ of $(a, b)$.

We transform this last condition as follows:

Lemma I. If $\beta_{n}$ is a sequence of functions uniformly of bounded variation such that

$$
\lim _{n} \int_{a_{1}}^{b_{1}} \beta_{n} d x=0
$$

for every subinterval $\left(a_{1}, b_{1}\right)$ of $(a, b)$, and if $u_{n}$ is the greatest lower bound of $\left|\beta_{n}(x)\right|$ for $x$ on $(a, b)$, then $\lim _{n} u_{n}=0$.

* Loc. cit., p. 283. See also Radon: Wiener Sitzungsberichte, vol. 122 (IIa), p. 1377, and Fischer, this Bulletin, vol. 27 (1920), p. 12. 
If $\lim _{n} u_{n}$ is not zero, then there will exist an $e>0$ and a subsequence $u_{n_{m}}$ of $u_{n}$ such that $u_{n_{m}}>e$ for every $n_{m}$, i.e., for every $n_{m}$ and every $x$ we have

$$
\left|\beta_{n_{m}}(x)\right|>e .
$$

Since the $\beta_{n_{m}}$ form a compact set, there exists a subsequence $\beta_{k}{ }^{\prime}$ approaching a limiting function $\beta$ also of bounded variation. This function $\beta$ will obviously be such that $|\beta(x)| \geqq e$ for every $x$ of $(a, b)$. Consequently there exists a subinterval $\left(a_{1}, b_{1}\right)$ of $(a, b)$ such that either

$$
\beta(x) \leqq-e \quad \text { or } \quad \beta(x) \geqq e
$$

for every $x$ of $\left(a_{1}, b_{1}\right)$, i.e.,

$$
\left|\int_{a_{1}}^{b_{1}} \beta(x) d x\right|>e\left(b_{1}-a_{1}\right) .
$$

But the $\beta_{k}{ }^{\prime}$ are uniformly bounded. Hence*

$$
\lim _{k} \int_{a_{1}}^{b_{1}} \beta_{k}{ }^{\prime}(x) d x=\int_{a_{1}}^{b_{1}} \beta(x) d x,
$$

which is not zero. Then we have reached a contradiction to the hypothesis of the lemma.

A direct consequence of this lemma is:

LEMMA II. If $\beta_{n}$ is a set of functions uniformly of bounded variation such that

$$
\lim _{n} \int_{a_{1}}^{b_{1}} \beta_{n}(x) d x=0
$$

for every subinterval $\left(a_{1}, b_{1}\right)$ of $(a, b)$, then in every subinterval $\left(a_{1}, b_{1}\right)$ of $(a, b)$ there exists a sequence of points $x_{n}$ such that

$$
\lim _{n} \beta_{n}\left(x_{n}\right)=0 .
$$

For, any subinterval $\left(a_{1}, b_{1}\right)$ of $(a, b)$ may replace $(a, b)$ in the hypothesis of Lemma I. Moreover, since $\lim _{n} u_{n}=0$, it follows that there will exist a point $x_{n}$ such that

i.e., $\lim _{n} \beta_{n}\left(x_{n}\right)=0$.

$$
\left|\beta_{n}\left(x_{n}\right)\right| \leqq u_{n}+\frac{1}{n},
$$

The conclusion of this lemma together with the fact that the $\alpha_{n}$ are uniformly of bounded variation is also sufficient for the convergence under consideration, i.e., we have:

* Cf. Lebesgue, Leçons sur l'Intégration, p. 114. 
Theorem. Necessary and sufficient conditions that the limit $\lim _{n} \int_{a}^{b} f d \alpha_{n}$ exist for every $f$ of $F$ are that

(1) the $\alpha_{n}$ be uniformly of bounded variation,

(2) there exist a function $\alpha$ of bounded variation such that if

$$
\beta_{n}(x)=\alpha_{n}(x)-\alpha_{n}(a)-\alpha(x)+\alpha(a),
$$

then

(a) $\lim _{n} \beta_{n}(b)=0$,

(b) in every subinterval $\left(a_{1}, b_{1}\right)$ of $(a, b)$ there exists a set of points $x_{1}, \cdots x_{n}, \cdots$ such that

$$
\lim _{n} \beta_{n}\left(x_{n}\right)=0 .
$$

The function $\alpha$ of the theorem may be taken to be the function which is the limit of a subsequence of $\alpha_{n}(x)$.

To prove the sufficiency, we show that, under the hypotheses of the theorem, $\lim _{n} \mathcal{S} f d \beta_{n}=O$ for every function $f$ of $F$. From a theorem of Bray* it follows that

$$
\left|\mathcal{S} f d \beta_{n}-\sum_{i=1}^{m} f\left(\xi_{i}\right)\left[\beta_{n}\left(x_{i-1}\right)-\beta_{n}\left(x_{i}\right)\right]\right| \leqq O_{\delta} M,
$$

where $x_{0}=a, x_{1}, \cdots, x_{m-1}, x_{m}=b$ is a subdivision of $(a, b)$, $\xi_{i}$ lies in the interval $\left(x_{i-1}, x_{i}\right), O_{\delta}$ is the maximum oscillation of $f(x)$ in $\left(x_{i-1}, x_{i}\right)$ and $\mathcal{S}\left|d \beta_{n}\right| \leqq M$ for every $n$. Since $f$ is uniformly continuous in $(a, b)$, for every $e$, there will exist a $d_{e}$ such that if $\left|x_{i}-x_{i-1}\right| \leqq d_{e}$, then $O_{\delta} \leqq e / 2 M$. Take a subdivision of $(a, b)$ by the points $\xi_{1}=a, \xi_{2}, \cdots, \xi_{m}=b$ such that for every $i,\left|\xi_{i}-\xi_{i-1}\right| \leqq \frac{1}{2} d_{e}$. Let $4 d_{0}$ be less than the minimum of $\xi_{i}-\xi_{i-1}$. We apply the hypothesis of the theorem to the intervals $\left(\xi_{i}+d_{0}, \xi_{i+1}-d_{0}\right)$, i.e., select a set of points $x_{i, n}$ in each interval such that $\lim _{n} \beta_{n}\left(x_{i, n}\right)=0$. We take $x_{0, n}=a, x_{1, n}, \cdots, x_{m, n}=b$, as points of division. Then since these points are finite in number, we can find an $n_{e}$ such that if $n \geqq n_{e}$

$$
\left|\sum_{i=1}^{m} f\left(\xi_{i}\right)\left[\beta_{n}\left(x_{i, n}\right)-\beta_{n}\left(x_{i-1}, n\right)\right]\right| \leqq e / 2 .
$$

* Loc. cit., p. 179. 
Since we also have

$$
\left|\mathcal{S} f d \beta_{n}-\sum_{i=1}^{m} f\left(\xi_{i}\right)\left[\beta_{n}\left(x_{i, n}\right)-\beta_{n}\left(x_{i-1}, n\right)\right]\right| \leqq e / 2,
$$

we have for $n \geqq n_{e}$

$$
\left|\mathcal{S} f d \beta_{n}\right| \leqq e \quad \text { or } \quad \lim _{n} \mathcal{S} f d \beta_{n}=0 .
$$

The conditions of the theorem may be simplified, if we note that the converse of Lemma II holds in the following form.

Lemma II'. If $\beta_{n}$ is any set of functions uniformly of bounded variation, then a sufficient condition that $\lim _{n} \int_{a}^{x} \beta_{n} d x=0$ for every $x$ of $(a, b)$ is that in every subinterval $\left(a_{1}, b_{1}\right)$ of $(a, b)$ there exist a sequence of points $x_{1}, \cdots, x_{n}, \cdots$ such that $\lim _{n} \beta_{n}\left(x_{n}\right)=0$.

The proof of this can be made along the lines of the sufficiency proof above.

As a consequence our theorem may be stated as follows.

Necessary and sufficient conditions that $\lim _{n} \int f d \alpha_{n}$ exist for every $f$ of $F$ are that:

(1) the $\alpha_{n}$ be uniformly of bounded variation;

(2) there exist a function $\alpha$ of bounded variation such that if $\beta_{n}(x)=\alpha_{n}(x)-\alpha_{n}(a)-\alpha(x)+\alpha(a)$, then

$\lim _{n} \beta_{n}(b)=0, \quad$ and $\quad \lim _{n} \mathcal{S}_{a}^{x} \beta_{n} d x=0$ for every $x$.

In this form it contains the following theorem of Lebesgue* as a special case. Necessary and sufficient conditions that $\lim _{n} \int f \varphi_{n}=0$ for every $f$ of $F$, the $\varphi_{n}$ being summable on $(a, b)$, are:

(1) $\mathcal{S}\left|\varphi_{n}\right| d x$ be bounded as to $n$;

(2) (a) $\lim _{n} \int_{a}^{b} \varphi_{n} d x=0$;

(b) for every $y, \lim _{n} \int_{y}^{b}(y-x) \varphi_{n}(x) d x=0$.

This results from the above theorem if we put

$$
\beta_{n}(x)=\int_{a}^{x} \varphi_{n}(x) d x .
$$

The University of Michigan.

* Sur les intégrales singuliers, ANnales DE Toulouse, (3), vol. 1 (1909), p. 57. 